\title{
Autoavaliação de um programa de mestrado profissional na Amazônia
}

\author{
Self-assessment of a professional master's program in the Amazon \\ Autoevaluación de un programa de maestría profesional en la Amazonía
}

Victor Matheus Silva Maués ${ }^{1 *}$, Lindinalva Brasil Monte ${ }^{1}$, Tatiana da Silva Mendes ${ }^{1}$, Carlos Jefferson Santana de Souza ${ }^{1}$, Maria Alves Belém ${ }^{1}$, Camila Negrão Monteiro ${ }^{1}$, Lena Claudia Maia Alencar ${ }^{1}$, Valéria Regina Cavalcante dos Santos ${ }^{1}$, Creusa Barbosa dos Santos Trindade ${ }^{1}$, Pilar Maria de Oliveira Moraes ${ }^{1}$.

\begin{abstract}
RESUMO
Objetivo: Analisar os dados apresentados no relatório de autoavaliação de um programa de mestrado profissional na Amazônia. Métodos: Este estudo é uma revisão documental do banco de dados do Programa de Pós-Graduação em Gestão e Saúde na Amazônia, no período de agosto/2020 a setembro/2021, a partir de um Programa de Mestrado Profissional na Amazônia vinculado a um Hospital de Ensino. Os grupos de interesse foram divididos em: discentes, gestão institucional e docente. Resultados: No relatório foi observado, entre as turmas A, B e C, sobretudo, no âmbito acadêmico, gestão e científica e tecnológica, uma efetividade variando de $60 \%$ a $100 \%$. A nível administrativo e de qualidade, essa porcentagem diminui, sobretudo nas turmas B e C, com indiferença na faixa de $63,6 \%$ a $70 \%$ na turma C. No contexto na gestão institucional e corpo docente, todos os aspectos apresentaram relativamente aceitáveis, todavia, considerando isoladamente o corpo docente, remete-se um índice de falta de conhecimento sobre o projeto pedagógico, de 6,2\% a 7,1\% e, quanto ao acompanhamento do egresso, 21,4\% discordam, $14,3 \%$ indiferente e 7,1\% discordam totalmente. Conclusão: Foi possível verificar as demandas específicas de cada categoria, sobretudo em dimensões administrativas e de qualidade, expondo limitações nos diferentes aspectos analisados.
\end{abstract}

Palavras-chave: Autoavaliação, Gestão, Pós-graduação, Saúde pública.

\begin{abstract}
Objective: To analyze the data presented in the self-assessment report of a professional master's program in the Amazon. Methods: This study is a documentary review of the database of the Postgraduate Program in Management and Health in the Amazon, from August/2020 to September/2021, from a Professional Master's Program in the Amazon linked to a Hospital of Teaching. Interest groups were divided into: students, institutional management and professors. Results: In the report it was observed, among classes A, B and C, especially in the academic, management and scientific and technological fields, an effectiveness ranging from $60 \%$ to $100 \%$. At the administrative and quality level, this percentage decreases, especially in classes B and $\mathrm{C}$, with indifference in the range of $63.6 \%$ to $70 \%$ in class $\mathrm{C}$. In the context of institutional management and teaching staff, all aspects were relatively acceptable, however, considering the faculty alone, there is an index of lack of knowledge about the pedagogical project, from $6.2 \%$ to $7.1 \%$ and, regarding the follow-up of the graduate, $21.4 \%$ disagree, $14.3 \%$ indifferent and $7.1 \%$ totally disagree. Conclusion: It was possible to verify the specific demands of each category, especially in administrative and quality dimensions, exposing limitations in the different aspects analyzed.
\end{abstract}

Key words: Self-evaluation, Management, Postgraduate studies, Public health.

\section{RESUMEN}

Objetivo: Presentar los resultados de la autoevaluación de un programa de maestría profesional en la Amazonía. Métodos: Se trata de un estudio transversal y analítico, de septiembre/2020 a febrero/2021, basado en un Programa de Maestria Profesional en la Amazonía vinculado a un Hospital Docente. Los grupos de interés se dividieron en: estudiantes, gestion institucional y docentes. Resultados: Se observó, entre las clases A, B y C, sobre todo, en el ámbito académico, gerencial y científico y tecnológico, una efectividad que varía del $60 \%$ al $100 \%$, sin embargo, en el nivel administrativo y de calidad, este porcentaje disminuye, sobre

\footnotetext{
1 Fundação Santa Casa de Misericórdia do Pará, Belém - PA. *E-mail: victor.maues@santacasa.pa.gov.br
} 
todo en las clases B y C, con indiferencia en el rango del $63,6 \%$ al $70 \%$ en la clase C. En el contexto de la gestión institucional y el profesorado, todos los aspectos fueron relativamente aceptables, sin embargo, considerando al profesorado de forma aislada, un referente Se hace falta de conocimiento sobre el proyecto pedagógico, de $6.2 \%$ a $7.1 \% \mathrm{y}$, en cuanto al seguimiento del egresado, $21.4 \%$ en desacuerdo, $14.3 \%$ indiferente y $7.1 \%$ en total desacuerdo. Conclusión: Fue posible verificar las demandas específicas de cada categoría, especialmente en las dimensiones administrativa y de calidad, exponiendo limitaciones en los diferentes aspectos analizados.

Palabras clave: Autoevaluación, Administración, Posgraduación, Salud pública.

\section{INTRODUÇÃO}

A avaliação institucional é um processo contínuo adotado para que indivíduos assumam posição de protagonismo, seja no setor micro na relação professor/aluno ou no olhar macro, dos órgãos reguladores nas esferas de educação (MINISTÉRIO DA EDUCAÇÃO (MEC), 2017; MEC, 2019a). No Brasil, no âmbito do ensino superior, o MEC ampliou o conceito de avaliação à autoavaliação e discorre da necessidade do "olhar para dentro" fomentadas pelas Comissões Permanentes de Avaliação (CPA), assim, possibilitando uma perspectiva de avaliação para além dos agentes externos, promovendo a interação dos membros participativos direto com o processo de ensino (NISKIER C, 2021).

Assim, esse conceito permeou a Pós-Graduação, na busca de monitorar qualitativamente os processos educacionais, científicos e de planejamento das ações dos programas, buscando compreender as dificuldades e suas noções resolutivas (FERREIRA WS, 2015; NISKIER C, 2021).

Para Lima ACS, et al. (2018), autoavaliação caracteriza como uma prática regular e sistemática de revisão das ações em relação a um modelo de excelência, permitindo a reflexão e consolidação de programas (LIMA ACS, et al., 2018; MEC, 2019b). Assim, tornou-se necessário compreender a infraestrutura existente e opiniões dos envolvidos: discentes, docentes, coordenação e outras categorias fugindo dos limitados modos de avaliação anteriores que dificultam as soluções de problemas institucionais (ex. avaliações externas) (VITÓRIA MIC, et al., 2014; LIMA ACS, et al., 2018).

Nesse sentido, a Coordenação de Aperf eiçoamento do Ensino Superior (CAPES) vem definindo o sentindo de avaliação na busca de fazer com que haja garantia do protagonismo de todos os sujeitos no processo avaliativo, bem como de demonstrar à sociedade os impactos científicos e de melhorar as distorções regionais existentes (MEC, 2019b; LEITE D, et al., 2020).

Assim, o estudo visa fornecer uma construção de identidade do programa, bem como monitorar seu crescimento e desenvolvimento na perspectiva de cumprimento do seu papel, proporcionando clareza ao processo autoavaliativo e expor os resultados da análise da comissão dos programas. Portanto, em alinhamento as novas normativas da CAPES, o objetivo deste estudo é apresentar os resultados de autoavaliação de um programa de mestrado profissional na Amazônia.

\section{MÉTODOS}

Este estudo é uma revisão documental do banco de dados de um Programa de Pós-graduação em Gestão e Saúde na Amazônia, realizado com coleta indireta do relatório de autoavaliação do programa. A coleta de dados foi realizada no período de agosto a setembro de 2021, após parecer do CEP 4.946.866.

O relatório foi produzido em dezembro de 2020 por uma Comissão de Autoavaliação integrada pela Reitora do Programa (Diretora), membros do planejamento estratégico, assessoria de qualidade, docentes e discentes do programa. Sendo analisado por atores que compõe e/ou coadunam com o processo de gestão do mestrado, quais sejam: docentes, discentes e gestão institucional.

A pesquisa foi realizada em consulta ao relatório de autoavaliação do programa sendo estruturada em categorias, contemplando os eixos: Acadêmicos; administrativos; científicos e tecnológicos, gestão e qualidade, tomando como base os documentos: Planejamento Estratégico Institucional e Ficha de Avaliação da CAPES (2017). 
As análises das respostas foram discutidas de maneira conjunta, considerando o caráter holístico da avaliação e a proposta de diversificar os apontamentos à construção do Programa. Foram realizados 6 encontros entre os membros e, ao final, apresentada e aprovada a ata de reunião; para melhor análise dos dados obtidos, os cinco primeiros encontros foram reservados para discutir os questionários individualmente e, no sexto, foram analisadas as respostas contidas no relatório de maneira geral para que a avaliação final fosse cruzada.

Visando manter questões éticas, optou-se pornão identificaro ano das turmas nas análises das respostas, sendo identificados pelas letras "A", "B" e "C". Após a análise do relatório, foi encaminhado a Coordenação do Programa sugestões de estratégias de ação para melhorias e desenvolvimento do programa.

\section{RESULTADOS E DISCUSSÃO}

Observou-se que a comissão de autoavaliação partiu de princípios norteadores para o processo da autoavaliação definindo um ancoramento para o trabalho (Figura 1).

Figura 1 - Princípios norteadores da Autoavaliação extraídos da proposta de autoavaliação do Programa.

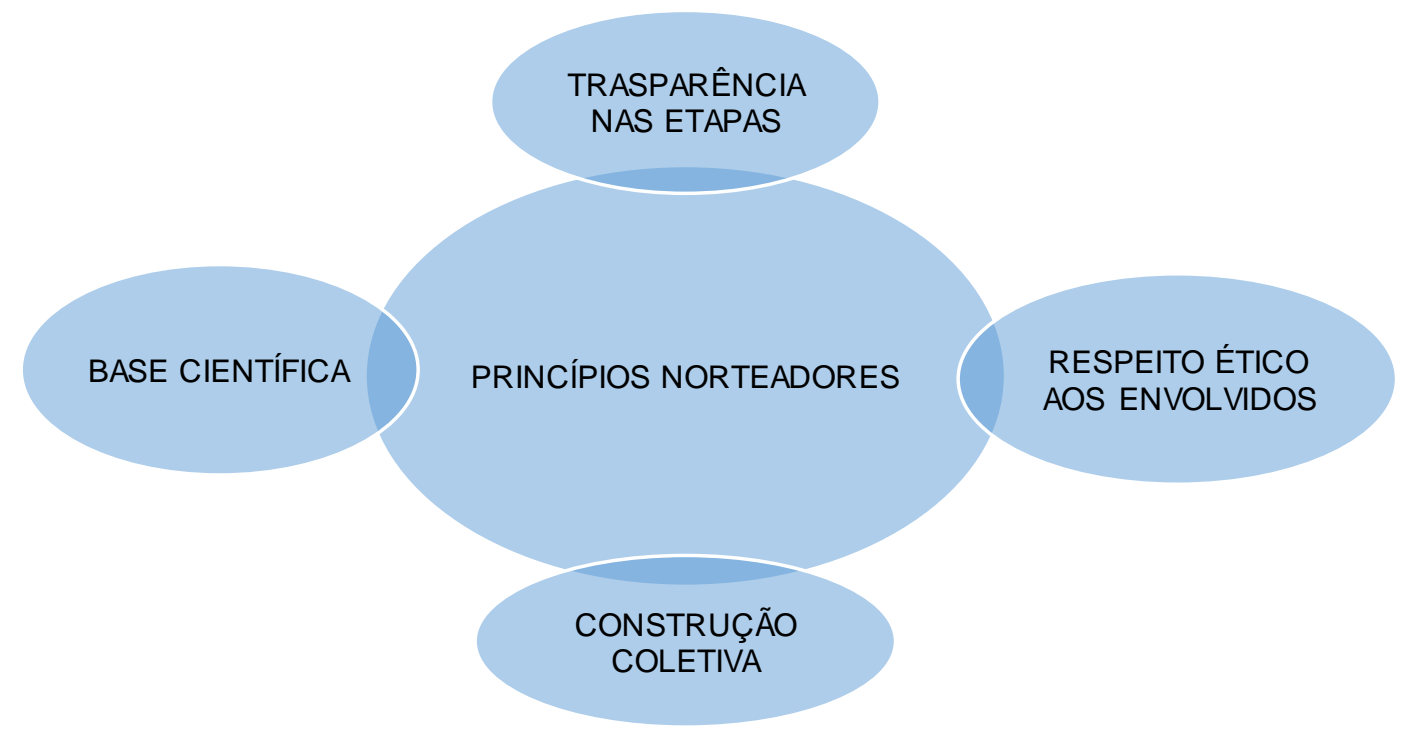

Fonte: Maués VMS, et al., 2021.

Pode-se visualizar que para avaliação, a comissão foi composta por membros do programa (corpo técnico e coordenação), discentes, docentes, setor de qualidade, planejamento e diretoria do ensino, pesquisa e extensão, assim, a expertise dos membros corroborou para uma avaliação holística. Para melhor compreensão, os resultados foram categorizados em: Perfil de atuação entre os discentes: Turma A; B; C; Perfil de atuação da Gestão Institucional e Perfil de atuação do Corpo Docente.

\section{Perfil de atuação entre os discentes: Turma $A$}

Composta por 12 mestrandos profissionais de instituições de saúde do estado do Pará, houve ef etiva participação de $83,33 \%$ dos discentes e, a partir das avaliações sobre as dimensões: acadêmica, administrativa, gestão, científica e tecnológica e qualidade; oi observado que, na dimensão acadêmica, referente ao conhecimento do projeto pedagógico, $90 \%$ responderam que concordam e, ao regimento do curso, $80 \%$. Quando detalhado sobre as ferramentas pedagógicas, 100\% disseram conhecer o plano de ensino e $90 \%$ pontua existir uma relação prática didático/pedagógico. No mais, quanto à produção científica, $90 \%$ concordaram à pertinência delas em relação à proposta do programa.

Referente as dimensões administrativas, a resolutividade da coordenação foi evidenciada em 90\%, a participação nas atividades desenvolvidas pelo programa e a avaliação da secretaria foi de $100 \%$ resolutiva em relação às demandas administrativas. Relativo à existência de acompanhamento do egresso à produção 
científica, tecnológica e seu desenvolvimento acadêmico profissional, $40 \%$ concordaram, $50 \%$ responderam indiferentes à questão e $10 \%$ discordaram.

Na dimensão científica e tecnológica, referente ao conhecimento sobre o planejamento estratégico do Hospital de ensino, 70\% concordaram que ele tem relação direta na produção científica e, na proposta do programa profissional, $100 \%$ concordaram, bem como $90 \%$ concederam que estivessem adequadas às linhas de pesquisa. Ademais, na dimensão da gestão, $100 \%$ responderam que conhecem o planejamento estratégico, e que os objetivos do programa estão $60 \%$ alinhado ao mesmo.

\section{Perfil de atuação entre os discentes: Turma B}

Composta por 13 mestrandos profissionais de instituições de saúde do Pará, havendo 80,33\% de ef etiva participação. Quando questionando a dimensão acadêmica, o percentual de concordância foi maior em questões de acesso as disciplinas (100\%), conhecimento do projeto pedagógico $(80 \%)$ e do regimento do programa $(90,9 \%)$. No mais, sobre a relação entre os conteúdos ministrados nas disciplinas e o contexto do local de trabalho, o percentual de indiferente é $36,4 \%$.

$\mathrm{Na}$ dimensão administrativa, houve manifesto de maior participação da coordenação e secretaria nas demandas apresentadas e, sobre resolutividade, o percentual de concordância concorda totalmente atinge $72,2 \%$, índice menor ao comparado as demais turmas.

Na dimensão científica e tecnológica, 100\% concordam que as produções científicas na linha de pesquisa integram as necessidades do programa, porém quando questionado de maneira específica aos produtos, o percentual chega em $81,8 \%$. Na dimensão da gestão, o conhecimento sobre o planejamento estratégico ainda é desconhecido (36,4\%). Na dimensão da qualidade, os discentes apontaram a necessidade de identificar os fluxos dos programas e ajustar os prazos de entrega do plano de ensino, plano aula, notas e frequência. Cabe ressaltar que esta turma teve sua entrada atrasada devido à pandemia, visto a suspensão das aulas e o decreto governamental que impossibilitou a realização de aulas presenciais.

\section{Perfil de atuação entre os discentes: Turma C}

Composta por 20 mestrandos, sendo profissionais das instituições de saúde do Pará e profissionais da Secretaria de Saúde Pública do Pará (SESPA), como forma de integrar a Secretaria ao programa e estimular possíveis parcerias de desenvolvimento científico e apoio tecnológico.

Na dimensão acadêmica, 90\% dos discentes afirmaram conhecer e/ou conhecer totalmente o projeto pedagógico, diminuindo para $80 \%$ sobre o regimento interno. Todos os discentes afirmam ter acesso ao plano de ensino e 10\% manifestaram ser indiferentes ao conhecimento sobre a relação didático pedagógica das disciplinas com o local de trabalho e se a produção científica atende as necessidades de um programa de mestrado profissional. Assim, os discentes mostraram conhecer e compreender os objetivos do programa e a organização da estrutura pedagógica, corroborando assim na construção de produtos coesos com a proposta do mestrado.

$\mathrm{Na}$ dimensão administrativa, o índice de indiferente foi maior quando questionado se existe acompanhamento de egresso à produção científica, tecnológica e o desenvolvimento acadêmico/profissional (63,6\%). Na dimensão científica e tecnológica, apontaram conhecer os impactos da produção científica para ampliar o alcance do programa e atualização constante do currículo lattes para alimentação da plataforma sucupira.

Referente à dimensão da gestão, apenas $50 \%$ concordam e/ou concordam totalmente que os servidores do Hospital de ensino conhecem os produtos do mestrado. Este dado pode estar relacionado ao fato de que o universo do hospital é grande, visto a parte administrativa e a assistência, que muitas vezes tem maior dificuldade em conhecer o que é realizado na área administrativa da instituição. Na dimensão da qualidade, o percentual de indiferentes foi maior quando perguntados se há estrutura adequada para orientação professor/aluno (30\%) e se há concordância quanto à definição dos fluxos internos do programa $(70 \%)$. 
Segundo Sias MAF (2018) e Rodrigues WFB (2018), a avaliação institucional deve ser um processo contínuo e dinâmico fundamentado na visão de criar, ajustar e qualificar os planos e ações da instituição, onde, devem estar sinalizados na proposta central de ensino e no Projeto Político-Pedagógico (PPP).

Dessa forma, o perfil de atuação dos discentes apresenta-se de forma positiva quanto aos fatores multidimensionais, todavia, de forma majoritária e recorrente entre as 3 turmas, se sobressai à dimensão administrativa como ponto importante a ser destacado, devido as maiores indiferenças frente aos fatores analisados, tendo como resoluções um olhar mais específico à turma, além da necessária uma participação mais ef etiva da coordenação e secretaria nas demandas apresentadas que, de acordo com Soares GF (2018), permitirá à gestão rede de ensino, no aspecto a possibilitar uma visão ampla do andamento das atividades em nível local, de forma a contribuir de forma significativa ao desempenho dos programas.

Destarte, segundo Soares SA (2014), em torno da complexidade em uma dimensão administrativa, é indispensável um planejamento cada vez mais comprometido, articulado e adequado aos processos envolvendo uma qualificação. Apesar das altas percentagens favoráveis às demais dimensões, não se alteram a constante necessidade das avaliações institucionais, sendo esse um instrumento para busca da qualidade educacional, concretização e ação-reflexão de todo o processo (LIMA C, 2017; SIAS MAF, et al., 2018).

Assim, a CAPES solicita que cada programa elabore sua autoavaliação, estimulando continuamente a integração entre o programa e a instituição da qual faz parte reiterando, dessa forma, a finalidade dos programas de pós-graduação além da produção de conhecimento, mas também uma formação participativa e que responde ao tópico ressaltado pela meta-avaliação. (BRASIL, 2019; LEITE D, et al., 2020).

No mais, outro aspecto recorrente, sobretudo na turma $\mathrm{B}$ e C, refere-se à dimensão da qualidade sendo apontado o fluxo interno das atividades do programa como problemática recorrente, devendo ser ajustado os prazos das atividades considerando o contexto pandêmico, além de exporuma adequação estrutural do corpo docente diante as orientações.

Nesse contexto, cabe salientar que nas turmas em questão houve interrupção das disciplinas, devido à pandemia do Covid-19, consequentemente, atraso no calendário acadêmico. Logo, para otimizar o calendário acadêmico, a reitoria do programa decidiu unir a turma $B$ ao calendário da turma $C$, tendo em vista o número de docentes permanentes do programa, além de otimizar as publicações dos discentes e a manutenção da qualidade do mestrado (FERREIRA LM, 2015).

Contudo, são notáveis as contribuições negativas dispostas ao discente em meio às mudanças dispostas. No mais, segundo a Capes (2019), para avaliação foi definido quesitos a ser avaliados, dentre eles, destacam se o perfil do corpo docente quanto à adequação e dedicação; distribuição das atividades, contribuição para atividades na graduação, compatibilidade com a proposta e produção intelectual devendo, portanto, constituir a orientação um instrumento importante à comunidade e para os programas de pós-graduação na procura de um padrão de excelência acadêmica nacional.

\section{Perfil de atuação da Gestão Institucional}

Participaram do questionário, o presidente da Instituição, a Diretora de Ensino, Pesquisa e Extensão, a Diretora de Planejamento, orçamento e gestão, o Diretor Financeiro e Administrativo e a Diretora Assistencial. Considerando que toda semana os diretores se reúnem, colocou-se como pauta a autoavaliação institucional e a necessidade das respostas do questionário. Assim, foi elaborado um texto apresentando o processo avaliativo, considerando as diretrizes preconizadas pela CAPES e obteve-se a participação de toda alta gestão da organização, sendo ponto positivo, visto a necessidade do olhar mais atento as demandas do Programa.

Na dimensão acadêmica, $62,5 \%$ concordam conhecer o projeto pedagógico e $62,5 \%$ afirmam conhecer os produtos gerados dentro das linhas de pesquisa que compõe o programa. Neste sentido, é importante incentivar a participação da alta gestão nos Workshops realizados pelo Mestrado, além de criar estratégias de divulgação dos produtos, de maneira interna e externa, visto que $12,5 \%$ dos gestores afirmaram não ter conhecimento sobre algum produto do mestrado que tenha sido utilizado como rotina do Hospital de ensino. 
No entanto, $100 \%$ dos gestores concordam que a produção científica tem impacto direto na produção do conhecimento e que há o alinhamento do programa ao planejamento estratégico institucional, bem como também afirmam que os produtos têm relevância econômica e social. No mais, metade dos gestores concorda que os servidores têm conhecimento dos produtos gerados no mestrado.

Assim, o Programa de Pós-graduação pode criar estratégias junto a Assessoria de Comunicação institucional para mapear o local de implantação dos produtos, que podem ser vistos a exemplo: o comitê de humanização, que fora criado um aplicativo para utilização pública; no centro cirúrgico, sendo criado checklist da cirurgia segura, entre outros. Por fim, $87,5 \%$ dos gestores afirmam concordar que há infraestrutura necessária ao atendimento docente/discente.

Em um programa de Pós-Graduação em Educação da Universidade do Estado de Mato Grosso, foi construída uma proposta de autoavaliação para concretização do mestrado condizente com a política da pósgraduação a nível nacional e internacional, nele, o perfil de gestão institucional envolve: acompanhamento das atividades; orientação; cumprimento dos critérios da CAPES; atendimento; solução dos problemas e tempo dedicado ao curso (UNEMAT, 2012).

Nesta perspectiva, considera-se importante criar estratégias de comunicação entre os discentes, docentes e produtos gerados do mestrado para com os líderes da organização, considerando que o foco do programa é a gestão e o planejamento em saúde e que os produtos trazem impacto direto para melhora dos processos organizacionais, redução de custos, segurança do paciente, desenvolvimento de competências profissionais, entre outros (SOARES GF, 2018; BRASIL, 2019).

Logo, reconhecendo que os programas de pós-graduação apresentam diferentes níveis de gestão institucional, é imprescindível as avaliações que permitem o diagnóstico oportuno, possibilitando ao gestor a percepção da sua atuação e a minimização de viés no local de trabalho. (CORRÊA NV, 2014; VITÓRIA MIC, et al., 2014).

\section{Perfil de atuação do Corpo Docente}

O Programa de Pós-graduação em Gestão e Saúde na Amazônia possui quatro docentes colaboradores e onze docentes permanentes, desses, 93,33\% participaram. Como forma de apresentar uma visão holística, as dimensões que foram avaliadas pelos discentes também foram utilizadas na avaliação dos docentes, assim, permitindo cruzar os dados e obter olhares diversos sob as mesmas dimensões apresentadas.

Na dimensão acadêmica, $86,7 \%$ afirmaram ter conhecimento sobre o projeto pedagógico. No entanto, considerando que este é o documento norteador que define o perfil do egresso, os parâmetros de avaliação, a descrição das disciplinas e créditos avaliativos, 7,1 \% dos docentes não concordam e 6,2\% são indiferentes ao conhecimento sobre o documento.

Assim, considera-se importante discutir ações para reduzir essa distância, seja em rodas de conversas, ou em reuniões entre coordenação e docentes, visto que, sem o conhecimento correto do projeto, a organização didática das disciplinas, a produção científica e outros aspectos gerais e específicos como: perfil de egresso, missão, visão, estrutura, grade curricular, ementas, poderão ser prejudicados (INEP, 2015; SIAS MAF, et al., 2018; BRASIL, 2019).

Sobre o regimento interno, $85,7 \%$ afirmaram que concordam em conhecê-lo, no entanto, o que chama atenção é o índice de 7,1\% que afirmam serem indiferentes a este conhecimento. Sobre a produção científica, $78,6 \%$ concordam e $7,1 \%$ concordam totalmente que possuem produções alinhadas a missão do programa. Porém, quando avaliado a percepção dos docentes quanto ao impacto da produção científica ao crescimento do programa, este valor diminuiu para $71,4 \%$ na concordância e sobe para $21,4 \%$ para discordo e $7,1 \%$ para indiferente.

Considerando que o programa em questão é interdisciplinar e que para isso precisa criar estratégias de interação na produção do conhecimento, $92,8 \%$ dos docentes avaliaram que utilizam do caráter interdisciplinar para produzir e orientar a pesquisa, isso evidencia que os docentes possuem conhecimento para orientação e construção do produto de pesquisa. 
Na dimensão administrativa, chama atenção a avaliação do docente quanto ao acompanhamento do egresso na sua produção científica, tecnológica no seu desenvolvimento acadêmico e profissional em relação à formação recebida, pois o relatório aponta que $21,4 \%$ discordam, $14,3 \%$ são indiferentes e, pela primeira vez nos questionários, $7,1 \%$ discordar totalmente.

Assim, ressalta-se que precisam ser criadas estratégias de alinhamento estratégico entre os objetivos do programa, à formação docente e a prática de orientação. Nesse aspecto, considera-se importante incluir na avaliação o tempo de permanência do docente no programa, sua visão em relação às orientações e a avaliação dos produtos após sua implantação, no mais, ressalta-se a necessidade de os docentes estimularem a produção científica e o mapeamento da ef etividade dos produtos após a implantação (LEBEL J e MACLEAN R, 2018; BRASIL, 2019).

Na dimensão científica e tecnológica, os docentes avaliaram que é necessário alinhar a produção científica as necessidades do programa, considerando que a percepção do impacto científico dos produtos gerados no mestrado pelos docentes ainda é de $78.6 \%$ de concordância. Referente ao alinhamento das disciplinas ministradas ao restante da grade curricular do curso, os docentes $92,8 \%$ dos docentes afirmam que concordam e/ou concordam totalmente, essa percepção é extremamente importante, considerando que a formação do mestrado precisa estar alinhada e numa escala de aquisição de conhecimentos.

Sobre o planejamento estratégico institucional, $35,7 \%$ dos docentes concordam em conhecer, $21,4 \%$ concordam totalmente, $21,4 \%$ discordam, 14,3 discordam totalmente e $7,2 \%$ são indiferentes ao conhecimento. Considerando que o hospital trabalha com foco no planejamento da organização e o programa de Mestrado está inserido nas ações estratégicas institucionais, a partir das atividades de extensão, qualificação e produção do conhecimento, torna-se necessário estimular a participação docente nas atividades de oficina do planejamento estratégico, com vistas a situar a importância do mestrado para o cumprimento da missão e alcance da visão institucional. Cabe ressaltar, que a instituição trabalha no conceito de excelência de maneira holística, onde todos os setores são protagonistas e precisam co-participar para esta construção.

Na dimensão da qualidade, quando questionados sobre as novas plataformas utilizadas, como: Google Meet $\AA$ e Google ClassRoom $\AA$, 85,7\% dos docentes expõem domínio da plataforma. Logo, considerando a pandemia, houve um ajuste na execução das disciplinas com a proposta de minimizar os impactos no percurso da formação dos discentes, assim, as aulas e a defesas das dissertações aconteceram de maneira presencial via remoto.

Esses dados são similares aos de Rodrigues WFB (2018) expressando que, apesar de receber uma capacitação docente para uso da plataforma, é necessário identificar as fragilidades existentes nesse modo de ensino-aprendizagem e estabelecer uma formação contínua aos ambientes virtuais de aprendizagem (VERHINE RE, 2019; RODRIGUES WFB, 2018; MENDONÇA AAS, 2020).

Considerando que o programa faz parte de uma instituição hospitalar de ensino, há uma particularidade na sua execução, visto que não existe em sua lei fundacional a possibilidade de cargo docente, fazendo com que técnicos que nele trabalham acumulem a função de docente em virtude de inexistência de dotação orçamentária para estes fins, a articulação como planejamento estratégico institucional, entre outros desafios que também desaguam no fato do mesmo ser executado na região norte do País, onde as assimetrias regionais persistem apesar dos grandes esforços, dado o percentual de mestres em relação à população total (LAVONEN J, 2017; MEC, 2019a; PPGGSA, 2020).

Após a avaliação, percebeu-se a necessidade de incluir outros grupos para avaliação, como a sociedade externa e assim avaliar os impactos da formação dos discentes no desenvolvimento laboral. No mais, a inclusão dos egressos do programa também possibilitará um monitoramento do papel social do programa e seus impactos, visto que por determinação da CAPES é obrigatório que até cinco anos a produção do aluno após a sua saída seja contabilizada para o programa; incluir também o corpo administrativo, assim, ampliar o caráter avaliativo para além dos aspectos já inclusos (MEC, 2019a). 


\section{CONCLUSÃO}

Apesar das concordâncias entre os envolvidos sobre as distintas dimensões, discordâncias e indiferenças foram recorrentes no público discente, sobretudo, nas turmas $B$ e $C$ referente às dimensões administrativas e qualidade, expondo limitações nos diferentes aspectos analisados, logo, considera a construção da pós graduação no Brasil uma tarefa complexa, dependente de muitos fatores internos e externos. Assim, no contexto da Amazônia, esse movimento ganha peculiaridades visto as distorções regionais e a necessidade de um olhar mais atento considerando as singularidades da região, portanto, se estabelece a necessidade de melhoria dos processos internos de gestão e planejamento estratégico, proporcionando o alcance dos trabalhos executados a nível administrativo, a qualidade e a ef etividade do programa, bem como na formação de recursos humanos dotados de conhecimento para intervir na sociedade.

\section{REFERÊNCIAS}

1. CORRÊA NV. Avaliação das estratégias de gestão da pós-graduação a partir do Programa de Excelência Acadêmica (PROEX). Dissertação (Mestrado em Educação em Ciências: Química da Vida e Saúde) - Universidade Federal do Rio Grande, Rio Grande, 2014.

2. FERREIRA LM. Mestrado profissional e seus desafios. Rev. Col. Bras. Cir. 2015;42(1): 9-13.

3. FERREIRA WS. A autoavaliação no âmbito da educação superior: o caso da Universidade Federal de Pernambuco. Recife, 2015.

4. INSTITUTO NACIONAL DE ESTUDOS E PESQUISAS EDUCACIONAIS (INEP). Anais dos seminários regionais sobre autoavaliação institucional e comissões próprias de avaliação (CPA). Brasília, 2015.Disponível em: Anais dos seminários regionais sobre autoavaliação institucional e Comissões Próprias de Avaliação(CPA) — Inep (www.gov.br). Acessado em: 3 de novembro de 2021

5. LAVONEN J. Governance decentralisation in education: Finnish innovation in education RED. Revista de Educación a Distancia, 2017; (53).

6. LEBEL J, MACLEAN R. A better measure of research from the global south. Comment. Nature, USA, 2018;559(4).

7. LEITE D, et al. A autoavaliação na Pós-Graduação (PG) como componente do processo avaliativo CAPES. Avaliação, Campinas; Sorocaba, 2020;25(2):339-353.

8. LIMA ACS, et al. GT Sobre a Autoavaliação De Programas De Pós-Graduação. Professores Membros - Portaria CAPES, 2018; 148:2-18.

9. MINISTÉRO DA EDUCAÇÃO (MEC). Coordenação de Aperfeiçoamento de Pessoal de Nível Superior - CAPES. Brasil. Portaria $n^{\circ} 389$, de 23 de março de 2017. Disponível em: http://cad.capes.gov.br/ato-administrativodetalhar?idAtoAdmElastic=241. Acessado em: 3 de novembro de 2021 .

10. MINISTÉRIO DA EDUCAÇÃO. Coordenação de Aperfeiçoamento de Pessoal de Nível Superior - CAPES. Brasil. Autoavaliação de Programas de Pós-Graduação: Grupo de Trabalho, 2019a. Disponível em: https://www.gov.br/capes/pt-br/centrais-de-conteudo/10062019-autoavaliacao-de-programas-de-pos-graduacao-pdf: Acesso em: 3 de novembro de 2021

11. MINISTÉRIO DA EDUCAÇÃO. Fundação Coordenação de Aperfeiçoamento de Pessoal de Nível Superior-CAPES. Brasil. GEOCAPES - Sistema de Informações Georreferenciadas. 2019b. Disponível em: http://portal.mec.gov.br/component/tags/tag/33975. Acesso em:3 de novembro de 2021.

12. MENDONÇA AAS. As novas tecnologias e a covid-19: o repensar da capacitação docente. CIET - Ressignificando a presencialidade, 2020.

13. NISKIER C. A educação não pode parar: coletânea de artigos. Brasília: ABMES, 2021.

14. SIAS MAF, et al. Avaliação Institucional: conceitos, objetivos, participação e implicações para a escola. RELACult Revista Latino-Americana de Estudos em Cultura e Sociedade, 2018.

15. SOARES SA. O equilíbrio entre as dimensões administrativa e pedagógica da gestão escolar como um desafio para diretores de escola: o caso da Superintendência Regional de Ensino de Pirapora/MG. Dissertação (mestrado profissional) - Universidade Federal de Juiz de Fora, Faculdade de Educação/CAEd. Programa de Pós-Graduação em Gestão e Avaliação da Educação Pública, 2014:160.

16. SOARES GF. Sistema de autoavaliação aplicado a programas de mestrado em rede, 2018.

17. RODRIGUES WFB, et al. Avaliação institucional e pedagógica do curso de pedagogia na perspectiva do discente no EAD. Revista humana e inovação, 2018;5(8).

18. UNIVERSIDADE DO ESTADO DE MATO GROSSO (UNEMAT). Proposta de Autoavaliação do Programa de PósGraduação em Educação - PPGEdu/UNEMAT/Cáceres: A auto-avaliação como instrumento auxiliar no planejamento, 2012. Disponível em: http://www.uffs.edu.br/campi/cerro-largo/cursos/mestradocl/mestrado-em-ensino-deciencias/legislacao-e-normas/repositorio-de-arquivos-1/plano-de-autoavaliacao-ppgec.pdf. Acesso em:20 de Outubro de 2021

19. VERHINE RE. O Sistema Nacional de Avaliação da Educação Superior após 14 anos: avanços e desafios. Educação superior e conhecimento no centenário da Reforma de Córdoba: novos olhares em contextos emergentes. Edi. PUCRS, 2019:79-94.

20. VITÓRIA MIC, et al. Autoavaliação dos programas de pós-graduação stricto sensu da PUCRS: Relato de experiência. In: SCARTON, A. M. et al. (Org.). Avaliação Institucional em IES Comunitárias. Porto Alegre:EDIPUCRS, $2014: 111-$ 126.

REAS | Vol.13(11) | DOI: https://doi.org/10.25248/REAS.e8788.2021 